ORIGINAL ARTICLE

\title{
Sentinel lymph node investigation in melanoma: detailed analysis of the yield from step sectioning and immunohistochemistry
}

\author{
H A Gietema, R J C L M Vuylsteke, I A de Jonge, P A M van Leeuwen, B G Molenkamp, \\ J R M van der Sijp, S Meijer, P J van Diest
}

J Clin Pathol 2004;57:618-620. doi: 10.1136/icp.2003.011742

See end of article for authors' affiliations

Correspondence to: Professor P A M van Leeuwen, VU University Medical Centre, Department of Surgical Oncology, PO Box 7057 $1007 \mathrm{MB}$ Amsterdam, The Netherlands;

pam.vleeuwen@vumc.nl

Accepted for publication 8 October 2003

\begin{abstract}
Aims: To evaluate in detail the extent to which step sectioning and immunohistochemical examination of sentinel lymph nodes (SLNs) in patients with melanoma reveal additional node positive patients, to arrive at a sensitive yet workable protocol for histopathological SLN examination.

Methods: The study comprised 29 patients with one or more positive SLN after a successful SLN procedure for clinical stage I/II melanoma. SLNs were lamellated into pieces of approximately $0.5 \mathrm{~cm}$ in size. One initial haematoxylin and eosin (H\&E) stained central cross section was made for each block. When negative, four step ribbons were cut at intervals of $250 \mu \mathrm{m}$. One section from each ribbon was stained with H\&E, and one was used for immunohistochemistry (IHC).

Results: When taking the cumulative total of detected metastases at level 5 as $100 \%$, the percentage of SLN positive patients increased from $79 \%, 83 \%, 83 \%, 90 \%$ to $93 \%$ in the H\&E sections through levels $1-5$, and with $\mathrm{IHC}$ these values were $83 \%, 86 \%, 90 \%, 97 \%$, and $100 \%$, respectively. One of six patients in whom metastases were detected at levels $2-5$ only had metastases in the subsequent additional lymph node dissection.

Conclusions: Multiple level sectioning of SLNs (five levels at $250 \mu \mathrm{m}$ intervals) and the use of IHC detects additional metastases up to the last level in melanoma SLNs. Although more levels of sectioning might increase the yield even further, this protocol ensures a reasonable workload for the pathologist with an acceptable sensitivity when compared with the published literature.
\end{abstract}

S ince the early 1990s a simple method has been available to detect and retrieve the first tumour draining lymph node, the so called sentinel lymph node (SLN). This is done with a radioactive tracer and blue dye, which are both injected around the tumour. ${ }^{1-3}$ During surgery, guided by the blue staining of the draining tissues, the radioactivity, and the preoperatively made lymphoscintigraphy, the SLN is removed and examined meticulously by the pathologist. When the SLN is identified successfully, the absence of tumour in this node predicts with a high degree of accuracy the absence of metastases in the remaining additional lymph nodes. ${ }^{4-7}$ The concept of the SLN as the first lymph node that drains a primary tumour has been validated extensively in the past few years. The SLN procedure had become the standard procedure for melanoma in many centres.

\begin{abstract}
"A compromise must be found between workload and sensitivity by limiting the degree of step sectioning and immunohistochemistry on multiple levels"
\end{abstract}

The reliability of the SLN procedure as an accurate staging procedure is dependent on the ability to identify the true SLN and the extent of histopathological examination of the SLN. Both are instrumental in limiting the false negative rate of the SLN procedure. Most metastatic deposits are thought to be found along the midplane of the node; therefore bisecting the node through the hilar plane, as recently described by Roberts and Cochran, may optimise the finding of metastases. ${ }^{8}$

Multiple level sectioning has been shown to increase the detection rate of SLN metastases in several studies. ${ }^{9}{ }^{10}$ However, complete serial sectioning would result in an unacceptable workload for the pathologist. Therefore, a compromise must be found between workload and sensitivity by limiting the degree of step sectioning and immunohistochemistry (IHC) on multiple levels. To date, no consensus exists on the most (cost)effective protocol for pathological analysis of SLNs. In fact, only a few studies have reported on the precise yield of each additional level of step sectioning and immunohistochemistry in breast ${ }^{8}$ and vulvar cancer, ${ }^{10}$ but no such study has yet been published for melanoma.

The traditional protocol used in our hospital includes step sectioning at $250 \mu \mathrm{m}$, as for other cancers. Although this protocol has yielded good clinical results for melanoma, it is not strictly evidence based, but rather experience based. Therefore, our study was performed to evaluate in detail the yield of multiple levels and the use of IHC for detecting metastases in SLNs, to arrive at an evidence based protocol for optimal SLN investigation.

\section{MATERIAL AND METHODS}

From a total of 189 consecutive patients with clinical stage I/II melanoma who underwent a successful SLN procedure from to January 1998 to September 2002, we identified 29 patients in whom one or more SLN proved to be positive. The mean age of the patients was 52 years (range, 35-79), and the mean Breslow thickness was $2.78 \mathrm{~mm}$ (range, 0.69-12).

We evaluated the extent of SLN processing required before these patients were found to be positive. All patients who were initially negative by haematoxylin and eosin (H\&E) on the first SLN section subsequently underwent standard multiple sectioning at $250 \mu \mathrm{m}$ intervals and staining with

Abbreviations: $\mathrm{H} \& \mathrm{E}$, haematoxylin and eosin; $\mathrm{IHC}$, immunohistochemistry; SLN, sentinel lymph node 
both H\&E and IHC. We assessed at which additional level patients who were initially found to be negative were subsequently converted to being SLN positive.

\section{Sentinel node biopsy}

The day before surgery, $40 \mathrm{MBq}$ of ${ }^{99 \mathrm{~m}} \mathrm{Tc}$ colloidal albumin was injected in two to four depots peritumorally. Lymphoscintigraphy was done to detect the presence, location, and number of focal accumulations.

Just before surgery, $0.5 \mathrm{ml}$ of $2.5 \%$ patent blue solution (Guerbet, Aulnay-sous Bois, France) was injected intracutaneously just around the scar on the site of the primary tumour or, when the tumour was still in situ, around the tumour. During surgery, focal tracer accumulations were localised using a handheld $\gamma$ probe (c-track; Carewise, Morgan Hill, California, USA). All hot and blue nodes were removed as SLNs. Furthermore, all radioactive nodes were biopsied until less than $10 \%$ of residual radioactivity, compared with the activity of the hottest SLN, remained in the lymph node basin. All patients underwent regional lymph node dissection after a positive SLN was detected.

\section{Tissue processing}

SLNs smaller than $0.5 \mathrm{~cm}$ were processed intact, those between 0.5 and $1 \mathrm{~cm}$ were halved, and SLNs larger than $1 \mathrm{~cm}$ were lamellated into pieces of approximately $0.5 \mathrm{~cm}$ in size. They were fixed in neutral buffered formaldehyde and embedded completely. One initial $4 \mu \mathrm{m}$ thick H\&E stained section was made from each block. When negative, an additional section was done at the first level for IHC and four step ribbons were cut at an interval of $250 \mu \mathrm{m}$. From these ribbons one section was stained with $\mathrm{H} \& \mathrm{E}$, one was used for IHC with S100, and one for HMB45 or Melan-A IHC (Dako A/D, Glostrup, Denmark). All slides were examined by the same pathologist (PJvD). All SLNs containing any cell compatible with melanoma cell morphology (and immunophenotype) were considered metastasis positive.

\section{RESULTS}

Of the 29 patients eventually found to have positive SLNs, 23 were positive on the first SLN section stained with H\&E. Interestingly, both patients with more than one positive SLN were found to have SLN metastases on the first H\&E stained section. With additional step sectioning and IHC in the remaining six patients, the percentage of SLN positive patients increased from $79 \%, 83 \%, 83 \%, 90 \%$, to $93 \%$ in the H\&E sections through levels $1-5$. With IHC these figures were $83 \%, 86 \%, 90 \%, 97 \%$, and $100 \%$, respectively. At the first level, IHC revealed one additional SLN positive patient and the additional levels 2-5 revealed metastases in one, one, two, and one more patient, respectively (table 1). Of the six patients with SLN metastases only detected at levels $2-5$, one had further metastases in the subsequent additional lymph node dissection.

Looking at all harvested nodes in these 29 SLN positive patients, 31 tumour positive SLNs were found. When taking

Table 1 Cumulative number of patients with melanoma sentinel node metastases found with haematoxylin \& eosin ( $\mathrm{H} \& \mathrm{E})$ and immunohistochemistry $(\mathrm{IHC})$ in each additional level ( $250 \mu \mathrm{m}$ intervals) in 29 patients

\begin{tabular}{lllll}
\hline & H\&E & $\%$ & IHC & $\%$ \\
\hline Level 1 & 23 & 79 & 24 & 83 \\
Level 2 & 24 & 83 & 25 & 86 \\
Level 3 & 24 & 83 & 26 & 90 \\
Level 4 & 26 & 90 & 28 & 97 \\
Level 5 & 27 & 93 & 29 & 100 \\
\hline
\end{tabular}

the cumulative total of detected metastases at level 5 as $100 \%$, the percentage of positive SLNs increased from $81 \%$, $84 \%, 84 \%, 90 \%$, to $94 \%$ in the H\&E sections through levels $1-5$. With IHC, the increase was from $84 \%, 87 \%, 90 \%, 97 \%$, to $100 \%$, respectively. The first level failed to detect metastases in five SLNs. Additional levels 2-5 yielded metastases in one, one, two, and one more positive SLN, respectively (table 2 ).

\section{DISCUSSION}

Because validation studies have shown high accuracy rates for the SLN procedure, ${ }^{11}$ it can now be used as a staging procedure without the need for completion lymph node dissection in patients with negative SLNs. ${ }^{4} 51112$

As long as the surgeon has performed a reliable SLN biopsy procedure, it is up to the pathologist to determine whether lymph node metastases are present or not.

In the days of routine elective lymph node dissection, it was shown that a large proportion of patients are converted from node negative to node positive with a more detailed histological examination of lymph nodes. ${ }^{13}{ }^{14}$ This has important consequences for the clinical management of these patients because, in general, lymph node positive patients will have a worse prognosis. ${ }^{2}$ For these reasons, the histopathological examination of SLNs tends to be even more elaborate, with the additional argument that this can be done with an acceptable workload for the pathologist because only a few nodes need to be examined this way.

Our study clearly shows that more patients are converted to node positive with each additional step of sectioning (at $250 \mu \mathrm{m}$ intervals), and that with each additional step IHC has a higher sensitivity than H\&E.

However, even with this intensive protocol not all nodal metastases will be detected. Such a $100 \%$ sensitivity can theoretically only be reached with complete serial sectioning of the SLN at $12 \mu \mathrm{m}$ intervals. ${ }^{10}$ In the study of Cook et al, reverse transcription polymerase chain reaction analysis slightly increased sensitivity for the detection of metastases, but at the cost of false positivity. ${ }^{14}$ Therefore, the place of this technique in routine diagnosis remains to be established. However, the question arises whether all metastatic cells need to be found. From a clinical point of view, only SLN metastases that are accompanied by second echelon or distant metastases need to be identified. Therefore, a method that has a sufficiently high clinical sensitivity with an acceptable workload for the pathologist needs to be defined.

Complete step sectioning of the SLN in patients with breast cancer has been done in only a few studies. For instance, Cserni serially sectioned the SLN up to extinction with 3-5 $\mu \mathrm{m}$ thick slices and examined every 10-20th level. ${ }^{15}$ In the final analysis, 15 of the 21 patients with metastases limited to the SLN were positive on the initial central cross section. Dowlatshahi et al performed complete serial sectioning at $250 \mu \mathrm{m}$ intervals and found that only six of 30 SLN positive patients were positive on the initial section examined by H\&E and IHC. ${ }^{16}$ This difference is remarkable, also in view of the

Table 2 Cumulative number of metastases found with haematoxylin \& eosin (H\&E) and immunohistochemistry $(\mathrm{IHC})$ in each additional level (250 $\mu \mathrm{m}$ intervals) in 31 cutaneous melanoma sentinel nodes

\begin{tabular}{lcccc}
\hline & H\&E & \% & IHC & $\%$ \\
\hline Level 1 & 25 & 81 & 26 & 84 \\
Level 2 & 26 & 84 & 27 & 87 \\
Level 3 & 26 & 84 & 28 & 90 \\
Level 4 & 28 & 90 & 30 & 97 \\
Level 5 & 29 & 94 & 31 & 100 \\
\hline
\end{tabular}


fact that Cserni's intervals were smaller and therefore it would be expected that a greater number of additional metastases would be found. The greater yield on the initial section in Cserni's study could be ascribed to the fact that they tried to cut the central cross section guided by the blue lymphatic vessel, which we did not, or the different biological properties of breast cancer and melanoma cells. In addition, the average size of the metastases may have been greater in patients in Cserni's study (68\% T2 tumours compared with $25 \%$ in the study by Dowlatshahi et al). No studies of complete sectioning of the SLN in patients with melanoma have been published, but the same results would be expected in melanoma.

"Immunohistochemistry facilitates the detection of single metastatic cells, and speeds up the screening of the sections dramatically"

Nevertheless, the fact remains that the use of IHC and additional step sectioning improves the detection rate of metastatic deposits. The question for practical purposes then remains: how many additional levels (and at what interval) need to be examined?

Turner et al examined 10 levels at $40 \mu \mathrm{m}$ intervals in 42 patients with SLN positive breast cancer in whom the initial H\&E section was negative. ${ }^{17}$ With the use of IHC, the first two levels of SLN examination found additional metastases in all but one of these patients. Therefore, they concluded that the additional eight levels of examination did not significantly contribute to the detection of additional metastases. However, with 10 levels at $40 \mu \mathrm{m}$ intervals, only $400 \mu \mathrm{m}$ of the entire SLN slice (up to $5000 \mu \mathrm{m}$ in thickness) is examined, which is unlikely to be sufficient for the detection of all metastases. Rather than taking many sections at small intervals, it may be more efficient to take fewer sections at larger step intervals.

Cook et al showed that in different patient groups, the percentage of SLN positive patients with IHC protocols involving only bivalving through the hilar plane, two additional levels with $50 \mu \mathrm{m}$ interval, and four additional levels at $50 \mu \mathrm{m}$ were $17.8 \%, 25.2 \%$, and $33.8 \%$, respectively. However, no direct evaluation of the contribution of each additional level was provided.

Our present study shows in detail the yield of step sectioning and IHC at five levels with an interval of $250 \mu \mathrm{m}$. In practice, this has proved to be an acceptable workload. Clearly, the yield increased with additional levels. The first level failed to detect metastases in five SLNs (16\% of the total number of metastases found). Additional levels 2-5 yielded metastases in one, one, two, and one more SLN, respectively. Thus, additional levels clearly reveal more metastases and even the fourth and fifth level together reveal 10\% additional metastases. Not surprisingly, the yield with IHC was higher than with H\&E only. IHC facilitates the detection of single metastatic cells, and speeds up the screening of the sections dramatically. Therefore, some investigators omit H\&E staining when IHC is performed. However, we prefer to make H\&E control sections because they are helpful in the detection of artefacts and benign inclusions.

These results are comparable to the number of additional metastases found by Torrenga et al in the SLNs of patients with breast cancer.9 They studied SLNs of patients with breast cancer processed in our institute according to the same protocol.

In conclusion, step sectioning of SLNs with IHC is very useful for finding the smallest metastases in SLNs of breast cancer that may be clinically relevant. Therefore, we propose step sectioning and IHC at four additional levels, separated by $250 \mu \mathrm{m}$ intervals, when the original H\&E section is tumour negative. Despite the fact that our protocol might still miss

\section{Take home messages}

- To optimise the detection of metastases in patients with melanoma, we propose step sectioning of sentinel lymph nodes and immunohistochemistry at four additional levels, separated by $250 \mu \mathrm{m}$ intervals, when the original haematoxylin and eosin section is tumour negative

- Although more levels of sectioning might increase the yield even further, this protocol ensures a reasonable workload for the pathologist with an acceptable sensitivity

some metastases we believe that this protocol ensures a reasonable workload for the pathologist with an acceptable sensitivity.

\section{ACKNOWLEDGEMENTS}

Supported by a grant from the Fritz Ahlqvist Foundation.

\section{Authors' affiliations}

H A Gietema, R J C L M Vuylsteke, I A de Jonge, P A M van Leeuwen,

B G Molenkamp, J R M van der Sijp, S Meijer, Department of Surgical Oncology, VU University Medical Centre, PO Box 7057, 1007 MB Amsterdam, The Netherlands

P J van Diest, Department of Pathology, VU University Medical Centre

\section{REFERENCES}

1 Morton DL, Wen DR, Wong JH, et al. Technical details of intraoperative lymphatic mapping for early stage melanoma. Arch Surg 1992; 127:392-9.

2 Statius Muller MG, van Leeuwen PAM, de Lange-De Klerk ES, et al. The sentinel lymph node status is an important factor for predicting clinical outcome in patients with stage I or II cutaneous melanoma. Cancer 2001;91:2401-8.

3 Veen H, van der Hoekstra OS, Paul MA, et al. Gamma probe-guided sentinel node biopsy to select patients with melanoma for lymphadenectomy. Br J Surg 1994;81:1769-70.

4 Bachter D, Michl C, Buchels $\mathrm{H}$, et al. The predictive value of the sentinel lymph node in malignant melanomas. Recent Results Cancer Res 2001;158:129-36.

5 Gershenwald JE, Thompson W, Mansfield PF, et al. Multi-institutional melanoma lymphatic mapping experience: the prognostic value of sentinel lymph node status in 612 stage I or II melanoma patients. J Clin Oncol 1999;17:976-83.

6 Jansen L, Nieweg OE, Peterse JL, et al. Reliability of sentinel lymph node biopsy for staging melanoma. Br J Surg 2000;87:484-9.

7 Statius Muller MG, Borgstein PJ, Piipers R, et al. Reliability of the sentinel node procedure in melanoma patients: analysis of failures after long-term follow-up. Ann Surg Oncol 2000;7:461-8.

8 Roberts AA, Cochran AJ. Current management of sentinel lymph nodes: perspectives from pathology. Curr Diagn Pathol 2003;9:199-210.

9 Torrenga H, Rahusen FD, Meijer S, et al. Sentinel node investigation in breast cancer: detailed analysis of the yield from step sectioning and immunohistochemistry. J Clin Pathol 2001;54:550-2.

10 van Diest PJ. Histopathological workup of sentinel lymph nodes: how much is enough? J Clin Pathol 1999;52:871-3.

11 Thompson JF, McCarthy WH, Bosch CM, et al. Sentinel lymph node status as an indicator of the presence of metastatic melanoma in regional lymph nodes. Melanoma Res 1995;5:255-60.

12 Reintgen D, Cruse CW, Wells K, et al. The orderly progression of melanoma nodal metastases. Ann Surg 1994;220:759-67.

13 Yu LL, Flotte TJ, Tanabe KK, et al. Detection of microscopic melanoma metastases in sentinel lymph nodes. Cancer 1999;86:617-27.

14 Cook MG, Green MA, Anderson B, et al. The development of optimal pathological assessment of sentinel lymph nodes for melanoma. J Pathol 2003;200:314-19.

15 Cserni G. Complete sectioning of axillary sentinel nodes in patients with breast cancer. Analysis of two different step sectioning and immunohistochemistry protocols in 246 patients. J Clin Pathol 2002;55:926-31.

16 Dowlatshahi K, Fan M, Bloom KJ, et al. Occult metastases in the sentinel lymph nodes of patients with early stage breast carcinoma: a preliminary study. Cancer 1999;86:990-6.

17 Turner RR, Ollila DW, Krasne DL, et al. Histopathologic validation of the sentinel lymph node hypothesis for breast carcinoma. Ann Surg 1997;226:271-6. 